\title{
Resümee und Ausblick
}

„Die Frage nach der gesellschaftlichen Verantwortung ist in den vergangenen Jahren eine zentrale Herausforderung für Unternehmen geworden. “1

\subsection{Zusammenfassung der gewonnenen Erkenntnisse}

Die vorliegende Dissertation befasste sich mit der grundlegenden Frage, wie Wirtschafts- und insbesondere Unternehmensethik vor dem Hintergrund einer kritischen Reflexion der Forschungstradition der Betriebswirtschaftslehre möglich, ja sogar höchst relevant ist und wie eine solche Ethik dann konzeptionell zu begründen wäre. Hierzu wurden im zweiten Kapitel die wissenschaftshistorischen sowie wissenschaftstheoretischen Grundlagen gelegt. Nach einem kompakten historischen Überblick über die Anfänge der Betriebswirtschaftslehre wurden die klassischen aber auch modernen Richtungsstreite in der Disziplin eruiert und gezeigt, warum sich die traditionelle BWL mit der Integration wirtschaftsethischer Forschung nach wie vor oft schwertut. Dabei wurden zwei zentrale, immer wieder aufkommende Kernargumente deutlich. Zum einen die Sorge, als vergleichbar junge akademische Disziplin keine Anerkennung im Kanon der anerkannten Wissenschaften zu finden, insofern man sich auf die „lediglich" diskursiv und nicht empirisch oder formal zu klärenden normativen Fragen einließe. Darüber hinaus hat gerade eine ethisch-normative Ausrichtung der BWL, insbesondere auch durch Erfahrungen normativer Theoriedeutungen

\footnotetext{
${ }^{1}$ Kleinfeld, A. / Schnurr, J. (2010), S. 286. Dabei kann auch mit SCHMALENBACH gerade für die freiheitliche Gesellschaft konstatiert werden: „In der freien Wirtschaft ist mit der Freiheit notwendigerweise ein Korrelat verbunden, die Verantwortung." (Schmalenbach, E. (1958), S. 24).
} 
in Zeiten des Nationalsozialismus, zu einer Entfremdung bzw. Skepsis gegenüber einer normativ orientierten Forschung und zu einer Hinwendung zu einer „sicheren“ und scheinbar wertfreien, mathematisch-idealtheoretisch begründeten Betriebswirtschaftslehre geführt. Neben diesem stark formalisierten, mikroökonomischen Ansatz von GUTENBERG hat sich im Sinne der Überlegungen von MELLEROWICZ aber auch als zweite, bis heute existierende Hauptströmung ein managementorientierter, verhaltenswissenschaftlich ausgerichteter Zweig der BWL etabliert, welcher im Sinne einer praktisch-normativen Disziplin auch intendiert, Handlungsempfehlungen zu geben, dabei allerdings bestimmte Ziele bzw. Zwecksetzungen (bspw. „Wertschöpfung“, „Gewinn“) traditionell als gegeben erachtet und lediglich die möglichen Mittel im Sinne der bestmöglichen Erreichung von bestimmten Zielen bzw. der Erfüllung eines gegebenen Zweckes evaluiert (Instrumentalfokus). Hierbei wurde jedoch deutlich, dass das klassisch zur Evaluation der Mittel angelegte ökonomische Prinzip alleine noch zu kurz greift, da ein reiner Rekurs auf den effizienten Mitteleinsatz mit vornehmlichem Vertrauen auf den legalistisch definierten Rahmen der Wirtschaftsordnung nicht mehr hinreichend legitimitätsstiftend wirkt. Vielmehr sind vor dem Hintergrund zunehmend kritischer Stakeholder und der verstärkten Einforderung unternehmerischer Verantwortung auch Ziele und Handlungen sowie deren weiteren Konsequenzen selbst zu reflektieren. Dies begründet folglich die Relevanz, sich aus einer originär betriebswirtschaftlichen Perspektive mit ethischen Fragestellungen zu beschäftigen. Mit diesen Ausführungen wurden dementsprechend die ersten beiden Forschungsfragen geklärt. So wurde erstens zum einen detailliert aus historischer Perspektive gezeigt, warum die BWL einer Integration ethischer Überlegungen noch häufig skeptisch gegenübertritt. Andererseits wurde zweitens aber auch gezeigt, dass eine Beschäftigung mit ethischen Fragestellungen aus genuin betriebswirtschaftlicher Perspektive als höchst relevant gelten kann, will die Betriebswirtschaftslehre dauerhaft erfolgreiches betriebliches Handeln unterstützen.

Basierend auf der herausgearbeiteten Relevanz einer vertieften wirtschaftsethischen Beschäftigung im Rahmen der betriebswirtschaftlichen Forschung befasste sich das dritte Kapitel dann detaillierter mit dem aktuellen Forschungsstand der Wirtschafts- und insbesondere auch der Unternehmensethik. Hierzu wurde zur terminologischen Präzision zuerst ein Überblick über einige Zentralbegriffe der Ethik gegeben. Darüber hinaus wurde die Ethik als Forschungsdisziplin genauer untersucht und die verschiedenen existierenden Forschungsrichtungen vorgestellt, wobei die Wirtschaftsethik als angewandte Ethik eingeordnet wurde. Hierauf aufbauend wurden dann die verschiedenen Ebenen der Wirtschaftsethik diskutiert, wobei die Unternehmensethik auf der Mesoebene, d. h. zwischen 
der Individualethik (Mikrofokus) und der gesellschaftlichen bzw. makroorientierten Rahmenordnungsethik verortet wurde. Nach Erarbeitung der grundlegenden Terminologien befasste sich die Arbeit eingehend mit dem aktuellen Forschungsstand, wobei insbesondere die detailliert ausgearbeiteten konzeptionellen Überlegungen des deutschsprachigen Raums vorgestellt und systematisiert wurden. Ferner wurde mit der angelsächsischen Forschungstradition eine angewandte wie pragmatisch fundierte Richtung aufgezeigt und der Forschungstradition des deutschsprachigen Raums methodologisch gegenübergestellt. Nach Erörterung des aktuellen wirtschaftsethischen Forschungsstandes erfolgte eine umfangreiche kritische Analyse, wobei, basierend auf den erarbeiteten Limitationen bisheriger Forschungsansätze, die Notwendigkeit einer neuen, genuin auf der Mesoebene zu verankernden Ethikkonzeption verdeutlicht wurde. Diese Ausführungen beantworteten folglich die dritte und vierte Forschungsfrage dieser Arbeit. So wurde, neben einer terminologischen Präzisierung einiger zentraler Begriffe der Ethik, systematisch der aktuelle Stand der wirtschaftsethischen Forschung reflektiert. Dabei konnte im Sinne der vierten Forschungsfrage detailliert gezeigt werden, dass bisher noch keine originäre Mesoethik existiert, welche ohne Rekurs auf klassische subjektphilosophische Überlegungen sowie ohne reine Appellation oder Rückfall auf traditionelle ökonomische Argumentationsmuster die Relevanz einer Unternehmensethik begründet. Für die weiteren Ausführungen sowie die fünfte Forschungsfrage wurden damit folgende Teilaspekte identifiziert: So steht erstens im Fokus, substantiiert die Relevanz einer originären mesoethischen Forschung herauszuarbeiten. Dabei ist also festzustellen, dass eine reine individual- oder ordoethische Verantwortungszuschreibung vor dem Hintergrund bestehender subjektphilosophischer Argumente, sowie insbesondere auch der heutigen globalisierten Wissensgesellschaft, zunehmend an ihre Grenzen stößt. Infolgedessen kommt der wirtschaftsethischen Forschung auf der Mesoebene ein besonderer, nicht-substituierbarer Status zu. Mit der Erkenntnis, dass Unternehmen in steigendem Maße als Verantwortungsträger auftreten sollten, ist zweitens zu klären, wie diese Verantwortungsübernahme ohne Rückgriff auf einen häufig evident unwirksamen reinen Verantwortungsappell sowie andererseits ohne Rückfall auf traditionelle ökonomische Argumentationsmuster im Sinne eines „Return on Ethics“, mit welchen jedoch die Gefahr eines Kategorienfehlers droht, konzeptionell fundiert zu begründen wäre. Drittens wurde dann die Notwendigkeit der Entwicklung einer originären Mesoethik ohne Rückgriff auf metaphysische und umstrittene Subjektparadigmen herausgearbeitet, wobei hierzu die Nutzung der Erkenntnisse der neueren Systemtheorie als vielversprechender Alternativansatz betont wurde. Schließlich stellte die kritische Reflexion auch die Relevanz einer Verbindung des konzeptionellen Forschungsfokus des 
deutschsprachigen Raums mit der Anwendungsorientierung der angelsächsischen Forschungstradition heraus.

Die Limitationen der bisherigen wirtschaftsethischen Forschung leiteten dann zum vierten Kapitel der Arbeit über, welches auf den identifizierten Forschungslücken aufbaute und die Entwicklung einer neuen subjektunabhängigen, analytischen Ethik auf systemtheoretischer Basis in den Fokus rückte. Vor dem Hintergrund einer zunehmend eingeforderten Verantwortungsübernahme durch Unternehmen befasste sich die Arbeit zuerst detaillierter mit dem Verantwortungsbegriff. Hierbei wurde nach einer kritischen Literaturanalyse ein vierstelliges Verantwortungsmodell vorgestellt, welches sich aus einem Subjekt als Verantwortungsträger, einem Objekt, welchem gegenüber Verantwortung zu tragen ist, der Instanz, welcher gegenüber sich zu verantworten ist sowie den zugrunde gelegten Normen zusammensetzt. Zum besseren Verständnis der Konstruktion dieses Verantwortungszusammenhangs wurde dann der Subjektbegriff detailliert konzeptionell gewürdigt. Als schillernder, facettenreicher Begriff wurde dieser zuerst einer etymologischen Analyse unterzogen, bevor die Arbeit die zentralen, in der Aufklärung begründeten Subjekteigenschaften herausstellte, welche als Basiseigenschaften seither klassisch zur Begründung der Verantwortungsfähigkeit von Individuen dienen. Ebenso wurde auch der Begriff der moralischen Person, welcher analog vor allem im angelsächsischen Schrifttum dominiert, einem kritischen Vergleich zum aufklärerischen Subjektbegriff unterzogen. Nach kritischer Rezeption des aufklärerischen Subjektbegriffs wurde deutlich, dass eine reine individualzentrierte Verantwortungszuschreibung zunehmend problematisch erscheint. Zudem haben gerade größere Unternehmen im Rahmen der Globalisierung in steigendem Maße Handlungspotentiale erhalten, während wiederum Einzelstaaten zunehmend Regulierungsmacht einbüßen, womit eine vornehmlich ordoethische Grundlegung ebenso ausscheidet. Die Begründung der Mesoverantwortung gelingt allerdings, wie die Arbeit verdeutlichte, trotz einiger Versuche in dieser Richtung, so z. B. von FRENCH, ebenfalls nicht überzeugend über einen Transfer des klassisch-aufklärerischen Subjektbegriffs auf die Mesoebene. Vielmehr ist hierzu ein anderer Begründungsansatz zu finden, welchen die Arbeit mittels Rückgriff auf die Überlegungen der neueren Systemtheorie aufzeigte. Hierzu wird das Unternehmen in einem neuartigen Ansatz als sozio-technisches System konzipiert, wobei die Erkenntnisse zur Fähigkeit der Autopoiesis bestimmter sozialer Systeme mit einem vertieften Verständnis technischer Systeme verknüpft wurden. Hierbei wurde deutlich, dass die Begründung von Unternehmensethik nicht metaphysisch über den Subjektbegriff erfolgen muss, es keine Moralappelle oder klassisch-ökonomische Überlegungen braucht, 
sondern sich im emergenten obersten Organisationsziel der Existenzsicherung findet. Die Beschäftigung mit normativen Aspekten liegt damit im wohlverstandenen Eigeninteresse des sozialen Systems, um seine Autopoiesis zu erhalten - und spezifisch im Sinne der Sicherung der Legitimität, welche zur wesentlichen Vorsteuergröße des normativen Managements im unternehmensethischen Sinne wird. Dabei zeigte die Arbeit insbesondere auch, dass eine rein strategisch-operative Ausrichtung, wie diese traditionell noch in der praktisch-normativen Betriebswirtschaftslehre vorherrschend ist, zur Legitimierung und damit Existenzsicherung vor dem Hintergrund zunehmend kritischer Stakeholder nicht mehr als ausreichend erachtet werden kann. So wurde die Relevanz einer originären normativen Managementebene deutlich, welcher institutionalisiert die Funktion des Aufbaus und Erhalts unternehmerischer Legitimität zukommt. Der in diesem Zusammenhang zentrale Begriff der Legitimität als Zustand sowie der Legitimierung als prozessuale Größe wurde ebenfalls detailliert gewürdigt und vor dem Hintergrund neoinstitutionalistischer Überlegungen die Bedeutung eines substantiierten normativen Managements herausgearbeitet. Nach dieser konzeptionellen Grundlagenforschung leitete die Arbeit dann im Sinne einer praktischen Nutzbarmachung der vorigen Überlegungen zur Entwicklung des Analytischen Frameworks über, welches sich zum Ziel setzt, das normative Management systematisch darin zu unterstützen, existierende Stakeholderansprüche abzuwägen und begründete, legitime Ansprüche nachvollziehbar in unternehmerischen Zielen und Handlungen $\mathrm{zu}$ implementieren. Hierbei geht es folglich um eine transparente Abwägung und Begründung, wobei mögliche Konflikte potentiell wohlbegründeter Ansprüche konzeptionell ebenso Beachtung finden, wie andere Restriktionen in der unternehmerischen Implementation und Durchführung. In diesem Zusammenhang wurde auch das zentrale Evaluationskriterium der Legitimität konzeptionell verfeinert, um der teilweise geäußerten Kritik einer rein deskriptiv-unkritischen Fundierung der Ethik entgegenzutreten. Im Rahmen eines neuartigen zweistufigen Ansatzes der Legitimität ist so nun erstmals, neben der Beachtung der faktisch vorherrschenden Moral, auch eine kritische wie systematische Evaluation anhand prinzipiell anerkannter Grundwerte deskriptiv sowie in Rekurs auf fundierte normative Ethikkonzeptionen ebenfalls ggf. ethisch-normativ integriert möglich. Die erste Stufe der Legitimitätsprüfung führt dabei zu einer notwendigen Legitimität, denn diese ist schlicht Voraussetzung, um in einer bestimmten Wertegemeinschaft dauerhaft erfolgreich die Geschäfte zu führen. Mit der zweiten Stufe der Legitimitätsprüfung sind diese Werte wiederum Gegenstand einer kritischen Reflexion, um so auch zu einer hinreichenden Legitimität zu gelangen. 
Mit den vorausgegangenen Ausführungen wurde folglich die fünfte Forschungsfrage beantwortet. So entwickelte die vorliegende Arbeit vor dem Hintergrund einer zunehmenden Relevanz der mesoethischen Verantwortungsübernahme eine Konzeption, welche auf systemtheoretischer Basis die Relevanz der Legitimitätssicherung aus systemischem Eigeninteresse begründen kann und somit kategorienkonform und ohne moralisierenden Appell operiert. Da aus genuin systemischer Perspektive argumentiert wird, gelingt es erstmalig, eine originäre Mesoethik zu konzipieren, bei welcher es sich nicht um eine letztlich verdeckte Individualethik oder der reinen Diskussion von Ethikfragen in Unternehmen handelt. Zudem zeigt diese Arbeit neben einer konzeptionell detaillierten Grundlegung auch eine Anschlussfähigkeit für die Praxis, welche wiederum mit dem Analytischen Framework verdeutlicht wurde. Aufgrund dessen, dass die Reflexion und Integration existierender Stakeholderansprüche üblicherweise ein komplexes Problem darstellt, wurde schließlich auch die Bedeutung von organisational institutionalisierten Sekundärwissensträgern herausgearbeitet, wobei ein besonderer Fokus auf den Implikationen für das Controlling lag. Dabei wurde aus Sicht der Entscheidungsunterstützung u. a. die Bedeutung einer kritischen Methoden- bzw. Instrumentenreflexion zur Legitimierung von Entscheidungen sowie darüber hinaus im Kontext der Entscheidungsbeeinflussung die bedeutsamen Erkenntnisse zur negativen Selektionswirkung klassischer Anreizsysteme herausgestellt, welche Individuen mit dunklen - und für den dauerhaften Erfolg des Unternehmens problematischen - Charaktereigenschaften anziehen. Mit diesen Ausführungen wurde so auch die sechste Forschungsfrage der Arbeit beantwortet. Abgeschlossen wurden die Ausführungen mit einigen Überlegungen zur Relevanz einer integren Organisationskultur als zentralem Einflussfaktor hinsichtlich der erfolgreichen Implementierung von unternehmensethischen Forschungserkenntnissen in der Praxis.

\subsection{Weitere Forschungsfragen}

Aufbauend auf die durch diese Arbeit gewonnenen Erkenntnisse ergeben sich einige weitere offene Forschungsfragen, die es zukünftig noch vertieft konzeptionell wie empirisch zu untersuchen gilt. Dies betrifft zuerst einmal die Operationalisierung des Legitimitätsbegriffs. So stellt dieser die zentrale normative Managementvariable aus Sicht der entwickelten Unternehmensethikkonzeption dar. Allerdings ist festzustellen, dass, neben einigen meist eher unsystematisch 
oder relativ domänenrestriktiv konzipierten Ansätzen ${ }^{2}$, bisher kaum Operationalisierungen existieren, welche sich der Entwicklung einer überzeugenden, allgemeinen, unternehmensübergreifend anwendbaren Skala gewidmet haben. ${ }^{3}$ Dies dürfte für die zukünftige Forschung höchst relevant zur Erforschung der legitimatorischen Konsequenzen von Unternehmensskandalen sowie der Wirksamkeit der ergriffenen Gegenmaßnahmen des Managements sein. Wie aufgezeigt wurde, beruht das im Rahmen dieser Arbeit entwickelte Konzept der Legitimität auf einem graduellen Ansatz. Folglich wäre zu prüfen, ab welchem Ausmaß ein kritischer Legitimitätspuffer unterschritten wird und welche weiteren Faktoren in existenziellen Krisen bedeutsam sind. Zu prüfen wäre auch, inwiefern bestimmte Items eine Grundlegitimität repräsentieren, welche im Sinne der „Mindestakzeptanz" auf jeden Fall vorhanden sein muss, während weitere, mit bestimmten Items operationalisierte Eigenschaften möglicherweise darüber hinausgehend zur Entstehung eines zusätzlichen Legitimitätspuffers beitragen. Die generell breite Anwendbarkeit des Konstrukts und die durch eine allgemeine Operationalisierung zu gewinnenden Erkenntnisse erscheinen für die weitere wirtschaftsethische Forschung besonders bedeutsam. Dabei wären ggf. auch kritisch verwandte Begrifflichkeiten wie Status und Reputation konzeptionell zu klären. Gerade bei letzterem Begriff wird teilweise eine scharfe Abgrenzung zur Legitimität intendiert. ${ }^{4} \mathrm{Zu}$ prüfen wäre hierbei allerdings, inwiefern bspw. der Reputationsbegriff nicht auch sinnvollerweise Legitimitätsurteile umfassen könnte, folglich also aus zwei distinkten Dimensionen besteht: eine normative Reputation (bspw. moralisch zu handeln) sowie eine technisch-instrumentelle Reputation (bspw. leistungsfähige Produkte bzw. Dienstleistungen zu kompetitiven Preisen herzustellen).

\footnotetext{
${ }^{2}$ Vgl. bspw. Elsbach, K. D. (1994), S. 86-87 zur Messung von Legitimität der Rinderzucht oder Foreman, P. / Whetten, D. A. (2002), S. 632-633 im Kontext ländlicher Kooperativen bzw. Genossenschaften. Darüber hinaus existieren auch in anderen Problemdomänen Operationalisierungsvorschläge, so z. B. zur Messung der Legitimität von Autorität, vgl. hierzu Tyler, T. R. (1997), S. 341-345. Vgl. auch die bisherigen Ansätze in Pollack, J. M. / Rutherford, M. W. / Nagy, B. G. (2012), S. 928-929; Vergne, J.-P. (2011), S. 496; Kostova, T. / Roth, K. (2002), S. 233.

${ }^{3}$ Eine Ausnahme bildet ein aktueller Beitrag von Bitektine, A. et al. (2020), der allerdings nur die soziopolitische sowie kognitive Legitimität operationalisiert, welche zudem, wie zuvor kritisch aufgezeigt wurde kaum als disjunkte Legitimitätsdimensionen aufgefasst werden können.

${ }^{4}$ Vgl. hierzu bspw. Bitektine, A. et al. (2020), S. 109-110.
} 
Im Kontext empirisch zu beobachtender Skandale und deren Auswirkung auf die unternehmerische Legitimität erscheint darüber hinaus eine vertiefte Verknüpfung mit der Forschung zum kollektiven Gedächtnis ${ }^{5}$ im normativen Kontext fruchtbar. Da hinreichend wirkungsstarke Legitimitätsbewertungen einer „kritischen Masse" an evaluierenden Stakeholdern bedürfen sowie auf dem Aggregat der Moral als faktisch vorherrschenden Werthaltungen beruhen, erscheint die Nutzbarmachung dieses Ansatzes für die wirtschaftsethische Forschung relevant. So wäre hier z. B. die Dynamik des Legitimitätsdiskurses zu untersuchen, also inwiefern bspw. skandalisierende Nachrichten sich in Netzwerken verbreiten und ab wann diese eine kritische Wirkung auf die Unternehmenslegitimität entfalten. Ebenfalls bedeutsam wäre in diesem Kontext ein vertieftes Verständnis hinsichtlich der Konsequenzen einflussreicher Stakeholder bzw. „Meinungsmacher“ im Legitimitätsdiskurs. Darüber hinaus wäre gerade im Zusammenhang mit Skandalen auch relevant, genauer zu verstehen, wann diese kollektiv vergessen werden, ob dies bspw. mittelfristig je nach Schwere überhaupt möglich ist ${ }^{6}$, und falls ja, in welchem Maße, sowie wiederum, inwiefern bestimmte Managementpraktiken im Legitimitätsdiskurs hilfreich sein können, um Skandale aus dem kollektiven (zumindest hinreichend präsenten) Bewusstsein zu entfernen.

Hinsichtlich der Ermittlung existierender Stakeholderansprüche sowie einer unternehmerischen Erfolgskontrolle aus Stakeholderperspektive wäre für die zukünftige Forschung auch die verstärkte Erforschung der Möglichkeiten von Big Data-Analysen im Rahmen der Industrie 4.0 sowie des Einsatzes von künstlicher Intelligenz vielversprechend. So könnten diese Technologien in verstärktem Maße genutzt werden, um die Ansprüche der Stakeholder besser zu verstehen sowie potentielle Legitimitätskrisen frühzeitig zu erkennen und die Möglichkeit der Offerierung eines diskursiven Einbezugs oder einer gezielten Ansprache sowie, falls diese Ansprüche wohlbegründet und realisierbar sind, einer verstärkten Integration in das unternehmerische Zielsystem vorzunehmen, um größere

\footnotetext{
${ }^{5}$ Die Forschungsrichtung des kollektiven Gedächtnisses beruht auf den Pionierarbeiten des französischen Soziologen MAURICE HALBWACHS in den 1920er Jahren. Vgl. hierzu klassisch Halbwachs, M. (1994), S. 146-221 sowie den Überblick bei Erll, A. (2017); Barash, J. A. (2016); Dimbath, O. / Heinlein, M. (2015); König, H. (2010); Berek, M. (2009); Pennebaker, J. W. / Banasik, B. L. (1997).

${ }^{6}$ So zeigt bspw. ähnlich die Forschung im Bereich der Reparatur von Vertrauen, dass das vorherige, vor einer Normverletzung vorherrschende Niveau an Vertrauen, insbesondere bei einer Täuschung, auch nach Entschuldigungen oder glaubwürdigen Gegenmaßnahmen nicht mehr erreicht wird. Vgl. hierzu Schweitzer, M. E. / Hershey, J. C. / Bradlow, E. T. (2006), S. 14-15.
} 
Legitimitätseinbußen durch öffentlichkeitswirksame Skandalisierungen zu unterbinden. Die durch diese neuen Technologien gewonnenen Erkenntnisse könnten dann - wiederum praktisch relevant - in einem normativen Management Cockpit transparent gemacht werden. Hierbei könnten auch normative „Red Flags“ definiert werden, die im Sinne der Komplexitätsreduktion einen hinreichend bedeutsamen Legitimitätsverlust indizieren könnten ${ }^{7}$, welcher bspw. wiederum mit positiv/negativ konnotierten Beiträgen bzw. Zustimmungswerten („Likes“, Weiterleitungen etc.) in Foren bzw. sozialen Netzwerken, aber auch mittels einer Analyse verschiedener Nachrichtenkanäle identifiziert werden könnte. In diesem Zusammenhang spielt folglich die Nutzung semantischer Analysen eine bedeutsame Rolle. In jedem Falle sind bei der Definition der notwendigen Maßgrößen die organisationalen Sekundärwissensträger als Spezialisten hinsichtlich der Ansprüche bestimmter Stakeholdergruppen vom Management in engem Maße einzubinden.

Zur weiteren institutionellen Absicherung dieser Maßnahmen könnte aus konzeptioneller Sicht zudem die Diskussion um die Sinnhaftigkeit einer rechtlichen Fundierung treten. Geht man davon aus, dass die betriebliche Tätigkeit immer auch ein gewisses Gefahrenpotential hinsichtlich der Erfüllung legitimer Ansprüche der Stakeholder induziert, so könnte ähnlich wie die existierende unionsweite Pflicht zur Nachhaltigkeitsberichterstattung von Großunternehmen ${ }^{8}$, für größere Unternehmen ebenso die Pflicht zur systematischen Reflexion und Berichterstattung, wie legitime Ansprüche in den unternehmerischen Zielbildungsprozessen sowie Handlungen hinreichend systematisch integriert werden, rechtlich verankert werden. In diesem Sinne wäre dann bspw. in einem „Ethics Report“ regelmäßig substantiiert zu begründen, wie eine solche systematische Anspruchsreflexion vorgenommen wurde, wobei wiederum auf die strukturierte Anwendung normativer Instrumente wie bspw. das Analytische Framework verwiesen werden könnte.

In diesem Zusammenhang erscheint folglich generell die Entwicklung zusätzlicher Managementinstrumente für die normative Ebene sinnvoll. Die vorliegende Arbeit hat hierbei besonders auf das Analytische Framework zur substantiierten wirtschaftsethischen Reflexion existierender Stakeholderansprüche fokussiert. Darüber hinaus ergeben sich jedoch vielfältige Anknüpfungspunkte für die Entwicklung weiterer normativer Instrumente. Interessant könnte hierbei bspw. die Entwicklung einer Balanced Value Card sein, welche explizit die normative Ebene gesellschaftlicher Werte mit den gewählten unternehmerischen Strategien

\footnotetext{
${ }^{7}$ Vgl. hierzu auch Lingnau, V. / Fuchs, F. (2019), S. 247.

${ }^{8}$ Vgl. RL 2014/95/EU (2014).
} 
(und ggf. auch operativen Zielen/Handlungen) verknüpft. Eine solche Darstellung könnte demnach nochmals helfen, die normative und strategische Ebene zu verzahnen, analog zur bereits existierenden Balanced Scorecard, welche bereits die strategische und operative Ebene verknüpft. Betrachtet man den überschaubaren aktuellen Forschungsstand hinsichtlich normativer Instrumente, gerade im deutschsprachigen Raum, so wäre auch dies gerade für eine praktisch-normativ orientierte BWL bedeutsam.

Hinsichtlich der zur Legitimierung getroffenen Maßnahmen lassen sich ebenfalls mehrere interessante empirische Forschungsfelder identifizieren. So könnte die zukünftige Forschung erheben, inwiefern sich bspw. mittelständische oder kleine Unternehmen von Großunternehmen bezüglich der allgemeinen Legitimierungsanforderungen unterscheiden. Neben einem direkten Kontakt zu den Stakeholdern dürfte dabei, wie zuvor schon hervorgehoben, vor allem bei großen Unternehmen oftmals die Tätigkeit in verschiedenen Kulturkreisen als Herausforderung existieren. Darüber hinaus wäre auch relevant, mehr über die Umsetzung normativer Ziele in konkrete Maßnahmen zu erfahren, also bspw. inwiefern die Unternehmensgröße und lokale versus globale Ausrichtung auch Konsequenzen auf die Legitimierungshandlungen sowie Legitimierungserfolge hat. Dabei wäre auch zu untersuchen, inwiefern sich ggf. im Falle von Legitimitätskrisen Verhaltensweisen bzw. Realitätsrekonstruktionen innerhalb verschiedener Unternehmensgrößen bzw. Branchen unterscheiden.

Aus konzeptioneller Perspektive wäre es zudem interessant, noch vertieft auf die normativen Steuerungsimplikationen einzugehen. So können, wie oben bereits aufgezeigt, insbesondere soziale Systeme nicht in einem trivialistischdeterministischen Sinne gesteuert, sondern lediglich perturbiert werden. Dies schränkt folglich die Beeinflussbarkeit anderer sozialer Subsysteme im organisationalen Zusammenhang ein und weist damit auch Konsequenzen in Bezug auf die Grenzen der Verantwortungsfähigkeit auf, welche das Management faktisch übernehmen kann. Darüber hinaus sind jedoch auch technische Systeme in zunehmendem Maße als komplex aufzufassen (z. B. globales Finanzsystem, Beschaffungsnetzwerke etc.). Die Implikationen einer solch begrenzten Steuerbarkeit auf die Übernahme von Verantwortung wären folglich für die weitere Forschung interessant. Hierbei könnte auch evaluiert werden, ob die Erkenntnis einer begrenzten Steuerungsfähigkeit bei (kleineren) Fehlentscheidungen unterer Managementebenen oder bei einem technischen Versagen als überzeugendes Argument gegenüber der Öffentlichkeit genutzt werden könnte, um negative Legitimitätskonsequenzen hinsichtlich des gesamten Unternehmens zu begrenzen.

Diese Arbeit zeigte zudem einige Implikationen für die Führungsunterstützung durch Sekundärwissensträger auf, wobei ein besonderer Fokus auf das 
Controlling gelegt wurde. Hierbei wurde neben einer kritischen Reflexion des eigenen intraorganisationalen Handelns sowie der eingesetzten Instrumente zur Entscheidungsunterstützung vor allem der negative Selektionseffekt durch traditionelle Anreizsysteme herausgestellt, welche Individuen mit psychopathischen Charaktereigenschaften anziehen. Diese bedeutsame Hypothese konnte grundsätzlich zum ersten Mal in einer Studie des Autors dieser Arbeit bestätigt werden. Weitere empirische Forschung könnte hierauf aufbauend noch detaillierter die negative Selektionswirkung extrinsischer Anreize untersuchen. So wäre z. B. zu klären, ob alle extrinsischen Incentives Individuen mit den genannten dunklen Charakterzügen gleichermaßen anziehen. In diesem Kontext könnte untersucht werden, ob bspw. nichtmonetäre extrinsische Incentives eine geringere Anziehung auf psychopathische Individuen ausüben, aber dennoch die meisten anderen Individuen zusätzlich motivieren. ${ }^{9}$ Hiermit könnte dann unter Rückgriff auf weitere intrinsisch wirkende Incentives (bspw. interessante Aufgaben) ein Portfolio unternehmerischer Anreize gestaltet werden, welche motivierend, aber in geringerem Maße negativ-selektiv wirken. ${ }^{10}$

Darüber hinaus könnte zur Gestaltung von psychopathieresistenten Organisationen im Sinne einer Schaffung von „Ethical Management Control Systems“ auch zukünftig verstärkt auf die aktuelle psychologische Forschung, welche sich zunehmend mit der Konzipierung des Gegenteils von Psychopathie beschäftigt, zurückgegriffen werden. So stellen etwa CANTER, YOUNGS und YANEvA das Prinzip „Kindness“, also ein integres, empathisches Wesen, dem Psychopathiekonstrukt gegenüber. ${ }^{11}$ Ähnlich, wenngleich mit anderer Terminologie, erachten WOODMASS und O'CONNOR „Compassionate Morality“ als das der Psychopathie entgegengesetzte Konstrukt. ${ }^{12}$ Schließlich entwickelten erst kürzlich KAUFMAN

\footnotetext{
${ }^{9}$ Darüber hinaus ist auch ein potentieller motivationaler Crowding Out zu berücksichtigen, also der Verdrängung intrinsischer Motivation durch extrinsische Incentives. Dieser Effekt hängt u. a. auch von der betreffenden Person und der Tätigkeit ab. So tritt ein durch extrinsische Anreize hervorgerufener Crowding-Out-Effekt vor allem bei Tätigkeiten auf, welche bereits durch ein hohes Maß an intrinsischer Motivation, d. h. durch Interesse oder Freude an der Tätigkeit selbst, gekennzeichnet sind. Vgl. Jost, P.-J. (2008), S. 499-502; North, K. / Güldenberg, S. (2008), S. 87 sowie die Ausführungen in Abschnitt 3.6.1. Extrinsische Anreize sind vor diesem Hintergrund daher eigentlich eher für monotone und weniger interessant empfundene Tätigkeiten zu begründen.

${ }^{10}$ So schlägt bspw. WIELAND neben ökonomischen Anreizen auch extrinsisch-moralische Anreize vor, wobei er als Beispiele die Achtung oder Anerkennung nennt. Vgl. Wieland, J. (2004b), S. 15-16.

${ }^{11}$ Vgl. Canter, D. / Youngs, D. / Yaneva, M. (2017), S. 17.

12 Vgl. Woodmass, K. / O’Connor, B. P. (2018), S. 259.
} 
ET AL. die s. g. „Helle Triade“, welche als Teilkonzepte den Humanismus, Kantianismus sowie den Glauben an die Menschlichkeit umfasst. ${ }^{13}$ All die genannten Konstrukte könnten sich zukünftig, neben der Gestaltung von Incentives, auch als hilfreich in der Entwicklung organisationaler Strukturen erweisen, welche für Individuen mit psychopathischen Charakterzügen nicht attraktiv erscheinen und damit die von IRTELLI und VINCENTI geforderte ,primary prevention“"14 unterstützen.

Des Weiteren wurde auch die Rolle der Kultur als wesentlicher Moderator bzw. Erfolgsfaktor bei der Integration unternehmensethischer Maßnahmen herausgearbeitet. Hierbei stellte die Arbeit die Bedeutung der Führungskräfte bei der Umsetzung unternehmensethischer Ansätze, wie bspw. dem Analytischen Framework, sowie die Relevanz einer integren Unternehmenskultur heraus. In diesem Zusammenhang wäre daher weitere konzeptionelle wie empirische Forschung, unter Rückgriff auf die existierenden Erkenntnisse über Möglichkeiten eines organisationalen ,cultural change“, hin zu einer für die Integration ethischer Maßnahmen förderlichen Kultur, sinnvoll. In Referenz auf die obigen Überlegungen hinsichtlich der Auswirkungen dunkler Charakterzüge in Führungspositionen könnte so auch der Korrumpierungseffekt durch psychopathische Führungskräfte künftig verstärkt untersucht werden und, sobald hinreichende Messinstrumente hierzu existieren, mit den Konsequenzen auf die unternehmerische Legitimität verknüpft werden.

Schließlich ergeben sich vor dem vielfach konstatierten Defizit einer normativen Diskursfähigkeit vieler Wirtschaftswissenschaftler auch Implikationen für die betriebswirtschaftliche Didaktik. So sind diese als Führungskräfte in der Praxis zunehmend gefordert, die unternehmerischen Ziele und Handlungen substantiiert und überzeugend begründen zu können, sind hierzu allerdings bisher akademisch nur in geringem Maße vorbereitet. In diesem Sinne erscheint eine vertiefte Beschäftigung mit wirtschaftsethischen Fragestellungen im betriebswirtschaftlichen Fächerkanon - gerade vor dem diskursethischen und analytischen Standpunkt der Arbeit - hochrelevant, um den Studierenden das Rüstzeug zu bieten, mit welchem diese zukünftig erfolgreiche Entscheidungen treffen können. Dabei geht es folglich, neben dem Ziel einer humanistischen Grundbildung, auch darum, dass diese zukünftigen Führungskräfte ihre Funktion besser, d. h. dauerhaft im Sinne des Unternehmens und seiner Stakeholder erfolgreicher, auszufüllen

${ }^{13}$ Vgl. Kaufman, S. B. et al. (2019), S. 7-9.

${ }^{14}$ Irtelli, F. / Vincenti, E. (2017), S. 193. 
vermögen ${ }^{15}$ - oder, um es mit PIES, HIELSCHER und BECKMANN nochmals pointiert hervorzuheben: „Wirtschafts- und Unternehmensethik hat nicht [nur, d. V.] den Anspruch, die Studierenden zu besseren Menschen zu erziehen, sondern sie hat das Ziel, die Studierenden zu besseren (erfolgreicheren) Managern auszubilden." 16 Folglich wäre eine verstärkte Aufnahme von ethischen Lehrinhalten in die Ausbildung der wirtschaftswissenschaftlichen Studierenden zentral, damit diese jederzeit mit guten Gründen einen Diskurs über die Sinnhaftigkeit des unternehmerischen Tuns durchführen und gegenüber allen Stakeholdern substantiiert Rede und Antwort stehen können.

Insgesamt hat die Arbeit die große Relevanz verdeutlicht, sich aus genuin betriebswirtschaftlicher Perspektive zukünftig noch verstärkt mit wirtschafts- und insbesondere auch unternehmensethischen Fragestellungen auseinanderzusetzen. Dabei war es stets das Ziel der Dissertation, nicht nur die Bedeutsamkeit einer Integration ethischer Fragestellungen in das betriebswirtschaftliche Forschungsgebäude hervorzuheben, sondern vielmehr auch die konzeptionellen Grundlagen zu legen, mit welchen eine solche Beschäftigung wissenschaftstheoretisch reflektiert sowie betriebswirtschaftlich-methodisch fundiert vor dem Hintergrund einer zunehmend globalisierten Wissensgesellschaft stattfinden kann. Letztlich kann, wie die Arbeit zeigt, unter Zuhilfenahme der neueren Systemtheorie ein strukturiertes Legitimitätsmanagement zur Begründung einer betriebswirtschaftlich sinnvoll zu integrierenden Unternehmensethikkonzeption herangezogen werden, welche die Tradition einer praktisch-normativen BWL weiterführt. Für die zukünftige Forschung sei die Hoffnung formuliert, dass die hier entwickelten Grundlagen einer systemtheoretisch fundierten, analytischen Konzeption in verstärktem Maße in den betriebswirtschaftlichen Diskurs eingehen und auch in der

${ }^{15}$ Darüber hinaus zeigen manche Studien im Kontext des s. g. Indoktrinationseffekts, dass eine dauerhafte unkritische Präsentation traditioneller idealtheoretischer ökonomischer Theorien auf die Werte der Studierenden einwirken kann, welche diese Inhalte verinnerlichen (vgl. bspw. Ifcher, J. / Zarghamee, H. (2018), S. 57-58; Etzioni, A. (2015), S. 231; Bauman, Y. / Rose, E. (2011), S. 324-325; Cipriani, G. P. / Lubian, D. / Zago, A. (2009), S. 463-466; Frank, R. H. / Gilovich, T. / Regan, D. T. (1993), S. 167-170). Hieraus resultiert dann die Gefahr, dass die Studierenden die axiomatischen Idealtheoreme fälschlich zur Lösung realer Fragestellungen, d. h. im praktisch-normativen Sinne, gebrauchen könnten. Zum Einfluss wirtschaftsethischer Bildung zur Verminderung professionellen Fehlverhaltens im Finanzberatungsbereich vgl. auch die Forschungsergebnisse bei Kowaleski, Z. T. / Sutherland, A. G. / Vetter, F. W. (2020), S. 165-169.

${ }^{16}$ Pies, I. / Hielscher, S. / Beckmann, M. (2009a), S. 324. Ähnlich formulieren auch PIES, BECKMANN und HIELSCHER: „The primary purpose of business ethics is, therefore, not to turn students into better people but into better managers." (Pies, I. / Beckmann, M. / Hielscher, S. (2010), S. 267). 
Praxis verstärkte Beachtung finden mögen. Ethik muss im unternehmerischen Kontext nicht appellativ angetragen werden, sie muss und darf allerdings auch nicht unreflektiert auf ökonomische „does it pay“-Argumente reduziert werden. Vielmehr geht es darum, die normativen Grundlagen zu legen, mit denen das einzelne Unternehmen, aber auch die gesamte Geschäftswelt dauerhaft in Einklang mit den legitimen Ansprüchen ihrer Stakeholder prosperieren kann. In diesem Sinne sei die Arbeit mit einem Zitat von SOLOMON geschlossen:

„My uncompromising thesis is that business ethics, rightly conceived, is just good business. This does not mean that virtue always prospers, but it does mean that the integrity of the corporation and of the individual within the corporation is the essential ingredient in the overall viability and vitality of the business world."17

Open Access Dieses Kapitel wird unter der Creative Commons Namensnennung 4.0 International Lizenz (http://creativecommons.org/licenses/by/4.0/deed.de) veröffentlicht, welche die Nutzung, Vervielfältigung, Bearbeitung, Verbreitung und Wiedergabe in jeglichem Medium und Format erlaubt, sofern Sie den/die ursprünglichen Autor(en) und die Quelle ordnungsgemäß nennen, einen Link zur Creative Commons Lizenz beifügen und angeben, ob Änderungen vorgenommen wurden.

Die in diesem Kapitel enthaltenen Bilder und sonstiges Drittmaterial unterliegen ebenfalls der genannten Creative Commons Lizenz, sofern sich aus der Abbildungslegende nichts anderes ergibt. Sofern das betreffende Material nicht unter der genannten Creative Commons Lizenz steht und die betreffende Handlung nicht nach gesetzlichen Vorschriften erlaubt ist, ist für die oben aufgeführten Weiterverwendungen des Materials die Einwilligung des jeweiligen Rechteinhabers einzuholen.

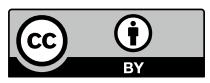

${ }^{17}$ Solomon, R. C. (1992b), S. 21. 